ISSN 2075-9827 e-ISSN 2313-0210

Carpathian Math. Publ. 2020, 12 (1), 107-110

doi:10.15330/cmp.12.1.107-110 http://www.journals.pnu.edu.ua/index.php/cmp

Карпатські матем. публ. 2020, Т.12, №1, С.107-110

MYtRofanOV M.A., RAVSKY A.V.

\title{
A NOTE ON APPROXIMATION OF CONTINUOUS FUNCTIONS ON NORMED SPACES
}

\begin{abstract}
Let $X$ be a real separable normed space $X$ admitting a separating polynomial. We prove that each continuous function from a subset $A$ of $X$ to a real Banach space can be uniformly approximated by restrictions to $A$ of functions, which are analytic on open subsets of $X$. Also we prove that each continuous function to a complex Banach space from a complex separable normed space, admitting a separating $*$-polynomial, can be uniformly approximated by $*$-analytic functions.

Key words and phrases: normed space, continuous function, analytic function, $*$-analytic function, uniform approximation, separating polynomial.
\end{abstract}

Pidstryhach Institute for Applied Problems of Mechanics and Mathematics, National Academy of Sciences of Ukraine, 3b Naukova str., 79060, Lviv, Ukraine

E-mail: mishmiterambler.ru(Mytrofanov M.A.), alexander.ravsky@uni-wuerzburg.de (Ravsky A.V.)

The first known result on uniform approximation of continuous functions was obtained by Weierstrass in 1885. Namely, he showed that any continuous real-valued function on a compact subset $K$ of a finitely dimensional real Euclidean space $X$ can be uniformly approximated by restrictions on $K$ of polynomials on $X$. For a compact subset $K$ of a finitely dimensional complex Euclidean space $X$ holds a counterpart of Stone-Weierstrass' theorem, according to which any continuous complex-valued function on $K$ can be approximated by elements of any algebra, containing restrictions on $K$ of polynomials on $X$ and their conjugated functions. A general direction of investigations is to try to extend these results to topological linear spaces. Most of the obtained results concern separable Banach spaces, although in the paper [4] the authors obtained partial positive results for separable Fréchet spaces. A negative result belongs to Nemirovskii and Semenov, who in [7] built a continuous real-valued function on the unit ball $K$ of the real space $\ell_{2}$, which cannot be uniformly approximated by restrictions onto $K$ of polynomials on $\ell_{2}$. This result showed that in order to uniformly approximate continuous functions on Banach spaces we need a bigger class of functions than polynomials. The following fundamental result was obtained by Kurzweil [3].

Theorem 1. Let $X$ be any separable real Banach space that admits a separating polynomial, $G$ be any open subset of $X$, and $F$ be any continuous map from $G$ to any real Banach space $Y$. Then for any $\varepsilon>0$ there exists an analytic map $H$ from $G$ to $Y$ such that $\|F(x)-H(x)\|<\varepsilon$ for all $x \in G$.

Separating polynomials were introduced in [3] and are considered in reviews [2] and [6]. In order to define them and to obtain a counterpart of Kurzweil's Theorem for a complex Banach space $X$, in paper [5] were introduced notions, which we adapt below for complex normed spaces $X$ and $Y$. 
A map $B_{k m}$ from $X^{k+m}$ to $Y$ is a map of type $(k, m)$ if $B_{k m}\left(x_{1}, \ldots, x_{k}, x_{k+1}, \ldots, x_{k+m}\right)$ is a nonzero map, which is $k$-linear with respect to $x_{i}, 1 \leq i \leq k$, and $m$-antilinear with respect to $x_{k+j}, 1 \leq j \leq m$.

Definition 1. A map $B_{n}: X^{n} \rightarrow Y$ is $*-n$-linear if

$$
B_{n}\left(x_{1}, \ldots, x_{k}, x_{k+1}, \ldots, x_{k+m}\right)=\sum_{k+m=n} c_{k m} B_{k m}\left(x_{1}, \ldots, x_{k}, x_{k+1}, \ldots, x_{k+m}\right),
$$

where for each $k$ and $m$ such that $k+m=n, B_{k m}$ is a map of type $(k, m)$ and $c_{k m}$ is either 0 or 1 , and at least one of $c_{k m}$ is non-zero.

Definition 2. A map $F_{n}: X \rightarrow Y$ is called an $n$-homogeneous $*$-polynomial if there exists a $*$-n-linear map $B_{n}: X^{n} \rightarrow Y$ such that $F_{n}(x)=B_{n}(x, \ldots, x)$ for all $x \in X$. Remark that $F_{0}$ is a constant map.

Definition 3. A map $F: X \rightarrow Y$ is a $*$-polynomial of degree $j$, if

$$
F=\sum_{n=0}^{j} F_{n},
$$

where $F_{n}$ is an $n$-homogeneous continuous $*$-polynomial for each $n$ and $F_{j} \neq 0$.

Definition 4. A map $H: X \rightarrow Y$ is $*$-analytic if every point $x \in X$ has a neighborhood $V$ such that

$$
H(x)=\sum_{n=0}^{\infty} F_{n}(x),
$$

where for each $n$ we have that $F_{n}$ is an $n$-homogeneous continuous $*$-polynomial and the series $\sum_{n=0}^{\infty} F_{n}(x)$ converges in $V$ uniformly with respect to the norm of the space $Y$.

Definition 5. Let $X$ be a complex (resp. real) normed space. A *-polynomial (resp. polynomial) $P: X \rightarrow \mathbb{C}$ (resp. to $\mathbb{R}$ ) is called a separating $*$-polynomial (resp. polynomial) if $P(0)=0$ and $\inf _{\|x\|=1} P(x)>0$.

Denote by $\widetilde{\mathcal{H}}(X, Y)$ the normed space of $*$-analytic functions from $X$ to $Y$.

Theorem 2 ([5]). Let $X$ be any separable complex Banach space that admits a separating *-polynomial, $Y$ be any complex Banach space, and $F: X \rightarrow Y$ be any continuous map. Then for any $\varepsilon>0$ there exists a map $H \in \widetilde{\mathcal{H}}(X, Y)$ such that $\|F(x)-H(x)\|<\varepsilon$ for all $x \in X$.

The aim of the present paper is to generalize Theorems 1 and 2 to normed spaces. To this end we need the following technical result.

Lemma 1. If a real normed space $X$ admits a separating polynomial $q$ then its completion $\widehat{X}$ admits a separating polynomial too.

Proof. Let $q=\sum_{i \in I} q_{i}$ be a sum of homogeneous polynomials $q_{i}$ on the space $X$. For each $i \in I$ there exists a polylinear form $h_{i}: X^{n_{i}} \rightarrow \mathbb{R}$ such that $q_{i}(x)=h_{i}(x, \ldots, x)$ for each $x \in X$. Since $h_{i}$ is a Lipschitz function on $X^{n_{i}}$, by [1, Theorem 4.3.17], it admits a continuous extension 
$\widehat{h}_{i}$ on the space $\widehat{X}^{n_{i}}$, which is polylinear by the polylinearity of $h_{i}$. The map $\widehat{q}_{i}: \widehat{X} \rightarrow \mathbb{R}$ defined as $\widehat{q}_{i}(x)=\widehat{h}_{i}(x, \ldots, x)$ for each $x \in \widehat{X}$ is an extension of the map $q_{i}$. Then the map $\widehat{q}=\sum_{i \in I} \widehat{q}_{i}$ is a continuous polynomial extension of the map $q$ onto the space $X$. It is easy to show that the unit sphere $S$ of the space $X$ is dense in the unit sphere $\widehat{S}$ of the space $\widehat{X}$. Therefore $\inf _{x \in \widehat{S}} \widehat{q}(x)=\inf _{x \in S} q(x)>0$, so $\widehat{q}$ is a separating polynomial for the space $\widehat{X}$.

Theorem 3. Let $X$ be a separable real normed space that admits a separating polynomial, $Y$ be a real Banach space, $A \subset X, f: A \rightarrow Y$ be a continuous function, and $\varepsilon>0$. Then there are an open set $A_{\varepsilon} \supset A$ of $X$ and an analytic function $f_{\varepsilon}: A_{\varepsilon} \rightarrow Y$ such that $\left\|f(x)-f_{\varepsilon}(x)\right\|<\varepsilon$ for all $x \in A$.

Proof. Let $\widehat{X}$ be a completion of $X$. We build a cover of the set $A$ by open in $\widehat{X}$ sets as follows. For each point $x \in A$ pick its neighborhood $O(x)$ open in $\widehat{X}$ such that $\left\|f\left(x^{\prime}\right)-f(x)\right\|<\varepsilon / 3$ for all $x^{\prime} \in O(x) \cap A$.

Put $\widehat{A}_{\varepsilon}=\bigcup_{x \in A} O(x)$. The topological space $\widehat{A}_{\varepsilon}$ is metrizable, and therefore paracompact, $[1,5.1 .3]$. Therefore, by $[1,5.1 .9]$ there is a locally finite partition $\left\{\varphi_{s}: s \in S\right\}$ of the unity, subordinated to the cover $\{O(x): x \in A\}$.

Now we construct an auxiliary function $f_{\varepsilon}^{\prime}: \widehat{A}_{\varepsilon} \rightarrow Y$. First, for each index $s \in S$ we define a real number $a_{S}$ as follows. If supp $\varphi_{s} \cap A \neq \varnothing$, then we pick an arbitrary point $x_{s} \in \operatorname{supp} \varphi_{s} \cap A$, and we put $a_{s}=f\left(x_{s}\right)$. Otherwise, we put $a_{s}=0$. Finally, put $f_{\varepsilon}^{\prime}=\sum_{s \in S} a_{s} \varphi_{s}$.

Let $x \in A$. Put $S_{x}=\left\{s \in S: x \in \operatorname{supp} \varphi_{s}\right\}$. Then $\sum_{s \in S_{x}} \varphi_{s}(x)=1$. Let $s \in S_{x}$ be any index. Thus there is an element $x_{0} \in A$ such that $x \in \operatorname{supp} \varphi_{s} \subset O\left(x_{0}\right)$. Hence $x_{s} \in O\left(x_{0}\right)$ and

$$
\left\|f(x)-a_{s}\right\|=\left\|f(x)-f\left(x_{s}\right)\right\| \leqslant\left\|f(x)-f\left(x_{0}\right)\right\|+\left\|f\left(x_{0}\right)-f\left(x_{s}\right)\right\|<2 \varepsilon / 3 .
$$

Then

$$
\begin{aligned}
\left\|f(x)-f_{\mathcal{E}}^{\prime}(x)\right\| & =\left\|f(x)-\sum_{s \in S} a_{s} \varphi_{s}(x)\right\|=\left\|\sum_{s \in S} f(x) \varphi_{s}(x)-\sum_{s \in S} a_{s} \varphi_{s}(x)\right\| \\
& =\left\|\sum_{s \in S_{x}} f(x) \varphi_{s}(x)-\sum_{s \in S_{x}} a_{S} \varphi_{s}(x)\right\| \leqslant \sum_{s \in S_{x}}\left\|f(x) \varphi_{s}(x)-a_{s} \varphi_{s}(x)\right\| \\
& =\sum_{s \in S_{x}}\left\|f(x)-a_{s}\right\| \varphi_{S}(x)<\sum_{s \in S_{x}}(2 \varepsilon / 3) \varphi_{S}(x)=2 \varepsilon / 3 .
\end{aligned}
$$

The function $f_{\varepsilon}^{\prime}$ is continuous on $\widehat{A}_{\mathcal{\varepsilon}}$ as a sum of a family of continuous functions with a locally finite family of supports.

By Lemma 1 , the space $\widehat{X}$ admits a separating polynomial. Therefore the space $X$ satisfies the conditions of Theorem 1 , so there exists a function $\widehat{f}_{\varepsilon}$ analytic on $\widehat{A}_{\varepsilon}$ such that $\left\|\widehat{f}_{\mathcal{\varepsilon}}(x)-f_{\mathcal{\varepsilon}}^{\prime}(x)\right\|<\varepsilon / 3$ for all $x \in \widehat{A}_{\mathcal{\varepsilon}}$. Then for all $x \in A$ we have

$$
\left\|f(x)-\widehat{f}_{\varepsilon}(x)\right\| \leqslant\left\|f(x)-f_{\varepsilon}^{\prime}(x)\right\|+\left\|f_{\varepsilon}^{\prime}(x)-\widehat{f}_{\varepsilon}(x)\right\|<\varepsilon .
$$

It remains to put $A_{\varepsilon}=\widehat{A}_{\varepsilon} \cap X$ and let $f_{\varepsilon}$ be the restriction of the map $\widehat{f}_{\varepsilon}$ to the set $A_{\varepsilon}$.

For a complex normed space $X$ we denote by $\widetilde{X}$ itself, considered as a real normed space, and by $\mathcal{H}(\widetilde{X}, Y)$ the real normed space of analytic functions from $\widetilde{X}$ to a Banach space $Y$. 
Theorem 4. Let $X$ be any separable complex normed space that admits a separating *-polynomial, $Y$ be any complex Banach space, and $F: X \rightarrow Y$ be any continuous map. Then for any $\varepsilon>0$ there exists a map $H \in \widetilde{\mathcal{H}}(X, Y)$ such that $\|F(x)-H(x)\|<\varepsilon$ for each $x \in X$.

Proof. The proof is almost identical to the proof of Theorem 4 from [5] with the following modifications. Instead of the application of Kurzweil's Theorem we apply Theorem 3. Instead of [5, Lemma 2] we use the fact (proof of which is similar to that of [5, Lemma 2]) that the identity map from a complex normed space $\widetilde{\mathcal{H}}(X, Y)$ to the real normed space $\mathcal{H}(\widetilde{X}, Y)$ is an isomorphism of real normed spaces.

\section{REFERENCES}

[1] Engelking R. General topology. Heldermann, Berlin, 1989.

[2] Gonzalo R., Jaramillo J.A. Separating polynomials on Banach spaces. Extracta Math. 1997, 12 (2) 145-164.

[3] Kurzweil J. On approximation in real Banach spaces. Studia Math. 1954, 14, $214-231$.

[4] Mytrofanov M.A., Ravsky A.V. Approximation of continuous functions on Frechet spaces. J. Math. Sci. (N.Y.) 2012, 185, 792-799. doi:10.1007/s10958-012-0961-6 (translation of Mat. Metodi Fiz.-Mekh. Polya 2011, 54 (3), 33-40. (in Ukrainian))

[5] Mitrofanov M.A. Approximation of continuous functions on complex Banach spaces. Math. Notes 2009, 86 (4), $530-$ 541. doi:10.1134/S0001434609090302 (translation of Mat. Zametki 2009, 86 (4), 557-570. doi:10.4213/mzm5161 (in Russian))

[6] Mytrofanov M.A. Separating polynomials, uniform analytical and separating functions. Carpathian Math. Publ. 2015, 7 (2), 197-208. doi:10.15330/cmp.7.2.197-208 (in Ukrainian)

[7] Nemirovskii A.S., Semenov S.M. On polynomial approximation of functions on Hilbert space. Math. USSR Sb. 1973, 21 (2), 255-277. doi:10.1070/SM1973v021n02ABEH002016 (translation of Math. Sb. 1973, 92 (134), 2 (10), 257-281. (in Russian))

Received 28.03.2020

Митрофанов М.А., Равський О.В. Про апроксисмацію неперервних функції в нормованих просторах // Карпатські матем. публ. — 2020. - Т.12, №1. - С. 107-110.

Нехай $X €$ дійсним сепарабельним нормованим простором, шо допускає відокремлювальний поліном. Показано, що непервні функції з підмножини $A$ в $X$ в дійсний банахів простір можуть бути рівномірно наближені аналітичними на відкритих підмножинах $X$. Також показано, що неперервні функції у комплексний банахів простір з комплексного сепарабельного нормованого простору, що допускає відокремлювальний *-поліном, можуть бути рівномірно наближені *-аналітичними функціями.

Ключові слова і фрази: нормований простір, неперервна функція, аналітична функція, *-аналітична функція, рівномірна апроксимація, відокремлювальний поліном. 